OPEN ACCESS

Edited by:

Anneliese Schrott-Fischer,

Innsbruck Medical University, Austria

Reviewed by:

Takeshi Tsutsumi,

Tokyo Medical and Dental

University, Japan

Franco Trabalzini,

University of Florence, Italy

*Correspondence:

Xiaowen Zhang

entxiaowen@163.com

Lijuan Song

entsong@163.com

†These authors have contributed equally to this work and share first authorship

Specialty section:

This article was submitted to Neuro-Otology,

a section of the journal

Frontiers in Neurology

Received: 05 October 2021 Accepted: 06 December 2021 Published: 05 January 2022

Citation:

Liu W, Chen G, Xie J, Liang T, Zhang C, Liao X, Liao W, Song $L$ and Zhang X (2022) A New Coordinate System for Magnetic Resonance Imaging of the Vestibular System.

Front. Neurol. 12:789887.

doi: 10.3389/fneur.2021.789887

\section{A New Coordinate System for Magnetic Resonance Imaging of the Vestibular System}

\author{
Weixing Liu ${ }^{\dagger}$, Gui Chen ${ }^{\dagger}$, Junyang Xie ${ }^{\dagger}$, Tianhao Liang ${ }^{\dagger}$, Chunyi Zhang, Xiao Liao, \\ Wenjing Liao, Lijuan Song * and Xiaowen Zhang *
}

State Key Laboratory of Respiratory Disease, Department of Otolaryngology-Head and Neck Surgery, The First Affiliated Hospital of Guangzhou Medical University, Guangzhou, China

Objectives: To develop and evaluate a new coordinate system for MRI of the vestibular system.

Methods: In this study, 53 internal auditory canal MRI and 78 temporal bone CT datasets were analyzed. Mimics Medical software version 21.0 was used to visualize and threedimensionally reconstruct the image data. We established a new coordinate system, named $\mathrm{W}-\mathrm{X}$, based on the center of the bilateral eyeballs and vertex of the bilateral superior semicircular canals. Using the $\mathrm{W}-\mathrm{X}$ coordinate system and Reid's coordinate system, we measured the orientations of the planes of the anterior semicircular canal (ASCC), the lateral semicircular canal (LSCC), and the posterior semicircular canal (PSCC).

Results: No significant differences between the angles measured using CT and MRI were found for any of the semicircular canal planes $(p>0.05)$. No statistical differences were found between the angles measured using Reid's coordinate system (CT) and the W-X coordinate system (MRI). The mean values of $\angle A S C C$ \& LSCC, LASCC \& PSCC, and $\angle$ LSCC \& PSCC were $84.67 \pm 5.76,94.21 \pm 3.81$, and $91.79 \pm 5.22$ degrees, respectively. The angle between the LSCC plane and the horizontal imaging plane was $15.64 \pm 3.92$ degrees, and the angle between the PSCC plane and the sagittal imaging plane was $48.79 \pm 4.46$ degrees.

Conclusion: A new $\mathrm{W}-\mathrm{X}$ coordinate system was developed for MRI studies of the vestibular system and can be used to measure the orientations of the semicircular canals.

Keywords: magnetic resonance imaging, coordinate system, semicircular canal, angle, orientation

\section{INTRODUCTION}

The annual prevalence of dizziness is approximately $11 \%$ in the United States $(1,2)$ and dysfunction of the vestibular system is one of the most common causes. The vestibular apparatus is very small and located on the deep side of the temporal bone (3). Two specific structures of the vestibular system, the otolith organs and semicircular canals, enable humans to perceive head rotation, angular acceleration, and spatial orientation (4). The orientations of the semicircular canals affect the sensitivity of the vestibule to angular acceleration $(5,6)$, and animals with more perpendicular canals encounter higher head angular velocity during locomotion (7). Thus, an accurate knowledge 
of the orientations of the semicircular canals is critical to understanding vestibular function and disease (8). The direction of the semicircular canals may determine the dynamics of endolymph flow, which in turn affects the sensory hair cells and ultimately the sensitivity of the tube system. Approximately 10$20 \%$ of patients with suspected benign paroxysmal positional vertigo (BPPV) cannot be accurately diagnosed or effectively treated using routine procedures $(9,10)$, which involve a series of head rotations at specific angles. For these subjects, the relative orientations of the semicircular canals may play a critical role in diagnostic complications and the effectiveness of treatment maneuvers (11).

Measuring the orientations of the semicircular canals requires a reliable three-dimensional (3D) coordinate system for the human brain. Frankfurt and Reid coordinate systems are commonly used in CT $(12,13)$, but these rely on bony landmarks that are difficult to identify accurately using MRI. Moreover, CT exposes subjects to radiation and cannot resolve the membranous labyrinth (14). Suzuki et al. used the total foot bifurcation point of the semicircular canals and the eyeball midpoint to determine the horizontal plane (HP) (15). Aoki et al. found that Reid's horizontal canal plane lies very close to the HP defined by Suzuki (16). However, different orientations of the semicircular canals have been reported using different coordinate systems $(11,17,18)$. In this study, we introduced and assessed a new coordinate system for MRI of the vestibular system.

\section{MATERIALS AND METHODS \\ Patients and Data Acquisition}

Imaging data were obtained from patients who underwent temporal bone high-resolution CT (Revolution 256-slice CT, GE Healthcare, IL, USA) or internal auditory canal MRI (Signa HDx 3.0T, GE Healthcare, IL, USA; Ingenia 3.0T, Philips, Netherlands) examinations at The First Affiliated Hospital of Guangzhou Medical University between December 1, 2018 and March 1, 2021. The scanning procedures followed standard temporal bone CT and inner ear MRI imaging protocols. CT $(120 \mathrm{kV}, 250 \mathrm{~mA}$, FOV: $320 \mathrm{~mm}$, matrix: $512 \times 512$, and slice thickness: $0.625 \mathrm{~mm}$ ), $3 \mathrm{D}$ fast-imaging using steady-state acquisition (FIESTA) MRI (GE, TR: $6.02 \mathrm{~ms}$, TE: $2.85 \mathrm{~ms}$, FOV: $180 \mathrm{~mm}$, matrix: 512 $\times$ 512, and slice thickness: $0.50 \mathrm{~mm}$ ), and T2W-3D-DRIVE MRI (Philips, TR: $2000 \mathrm{~ms}$, TE: $200 \mathrm{~ms}$, FOV: $150 \mathrm{~mm}$, matrix: $480 \times 480$, and slice thickness: $0.50 \mathrm{~mm}$ ) were performed independently. Inclusion criteria were the clear visualization of the anatomical structures of the cochlea and semicircular canals. The exclusion criterion was an inner ear deformity or superior semicircular canal dehiscence. The final dataset included 123 patients, 78 CT scans (male: 37, female: 41), and 53 MRI scans (GE, male: 35, female: 12; Philips, male: 2, female: 6). Both CT

Abbreviations: ASCC, anterior semicircular canal; PSCC, posterior semicircular canal; LSCC, lateral semicircular canal; HP, horizontal plane; SP, sagittal plane; FP, frontal plane; W-HP, W-X horizontal plane; R-HP, Reid's horizontal plane; F-HP, Frankfurt horizontal plane; S-HP, Suzuki's horizontal plane. and MRI data were available for 8 patients. The study protocol was reviewed and approved by The First Affiliated Hospital of Guangzhou Medical University review board, Guangzhou, Guangdong, China.

\section{Image Analysis and Coordinate Systems}

Digital Imaging and Communication in Medicine (DICOM) image files for CT and MRI data contain positioning data that define the spatial coordinate in the super-inferior, anteroposterior, and left-right directions. Mimics Medical software version 21.0 was used to view these images and reconstruct these images in 3D images. We used Reid's coordinate system for CT images (12) and a new W-X coordinate system for MR images.

Reid's coordinate system is based on anatomical landmarks independent of the head position of the subject during the CT examination. Reid's horizontal plane (R-HP) is defined to pass through the inferior margin of the two orbits and bilateral midpoint of external auditory canals. Reid's sagittal plane (R$\mathrm{SP}$ ) is defined as the plane that lies perpendicular to R-HP and connects the left-right orbital midpoint and the bilateral midpoint of the opening of external auditory canals. Reid's frontal plane (R-FP) is defined as the plane that lies perpendicular to both R-HP and R-SP and passes through the midpoint of the line connecting the tips of the bilateral midpoint of the external auditory canals (Figure 1).

The $\mathrm{W}-\mathrm{X}$ horizontal plane (W-HP) was defined as the plane passing through the center of the bilateral eyeballs and vertex of the bilateral superior semicircular canals. The W-X sagittal plane (W-SP) was defined as the plane that lies perpendicular to $\mathrm{W}-\mathrm{HP}$ and connects the center of the bilateral eyeball midpoint and the line connecting the tips of the vertex of the bilateral superior semicircular canals. The $\mathrm{W}-\mathrm{X}$ frontal plane (W-FP) was defined as the plane that lies perpendicular to both $\mathrm{W}$ $\mathrm{HP}$ and W-SP and passes through the midpoint of the line connecting the tips of the bilateral midpoint of external auditory canals (Figure 2).

In addition, we established and compared differences among the R-HP, W-HP, Frankfurt horizontal plane (F-HP), and Suzuki horizontal plane (S-HP) (Figure 3). F-HP is the plane passing through the bilateral vertex of the opening of the external auditory meatus and the left infraorbital edge point (17). SHP was defined as the plane passing through the center of the bilateral eyeballs and the bilateral bifurcations of the common crus (15).

\section{Angle Measurement}

The relative orientations of semicircular canals within the cranial base were defined as the angles between the standard sagittal plane (SP), horizontal plane (HP), and frontal plane (FP) and each canal plane (Figure 4). The anterior, posterior, and lateral semicircular canals were referred to as ASCC, PSCC, and LSCC, respectively. The "fit center line" tool of the Mimics Medical software was used to obtain the centerline and 3D coordinate $(x$, $y, z)$ of the center point of each semicircular canal. A multipoint least-squares fitting method was used to define the canal planes 

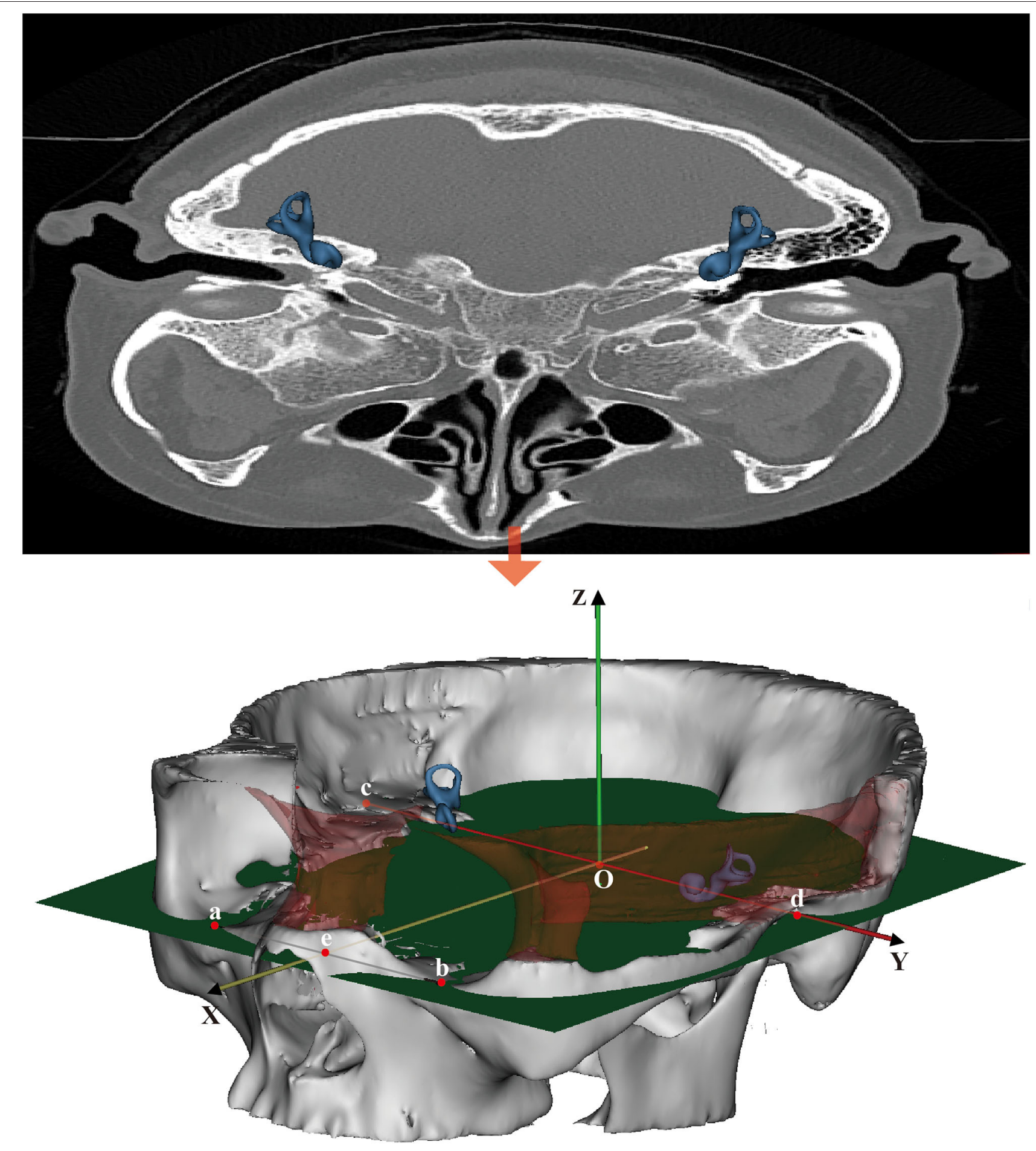

FIGURE 1 | Reid's coordinate system overlaid on a CT image. Points a and b are the bilateral midpoints of the inferior margin of the orbits. Points $c$ and $d$ are the bilateral midpoints of the external auditory canal. Point $\mathrm{e}$ is the midpoint of the line segment $\overline{a b}$. The origin of the coordinate system, point $\mathrm{O}$, is the midpoint of line segment $\overrightarrow{\mathrm{cd}}$. The $x$ - and $y$-axes are defined by the rays $\overrightarrow{\mathrm{Oe}}$ and $\overrightarrow{\mathrm{Od}}$, respectively, and the $z$-axis is perpendicular to $x$ and $y$ (+superior). The sagittal plane (SP) was defined as the plane that contains point $\mathrm{O}$ and is perpendicular to the $y$-axis. 


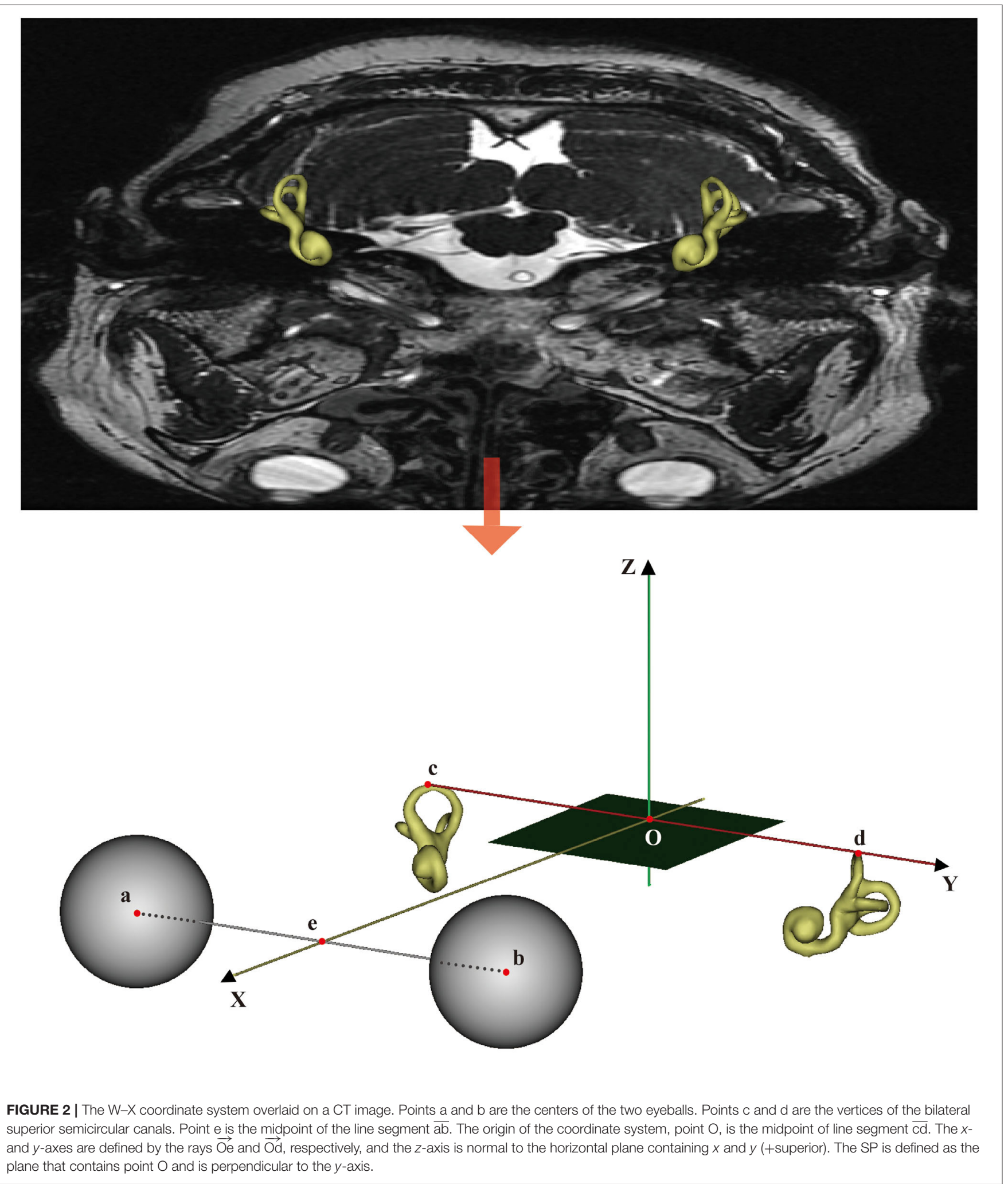

(20-40 center positions in each section) and four points of the W-HP (12). Coordinate values were imported into an in-house program written in the MATLAB (MathWorks Inc.,
Natick, MA, USA) programming language. Two investigators measured the relative orientations of the semicircular canals independently. 


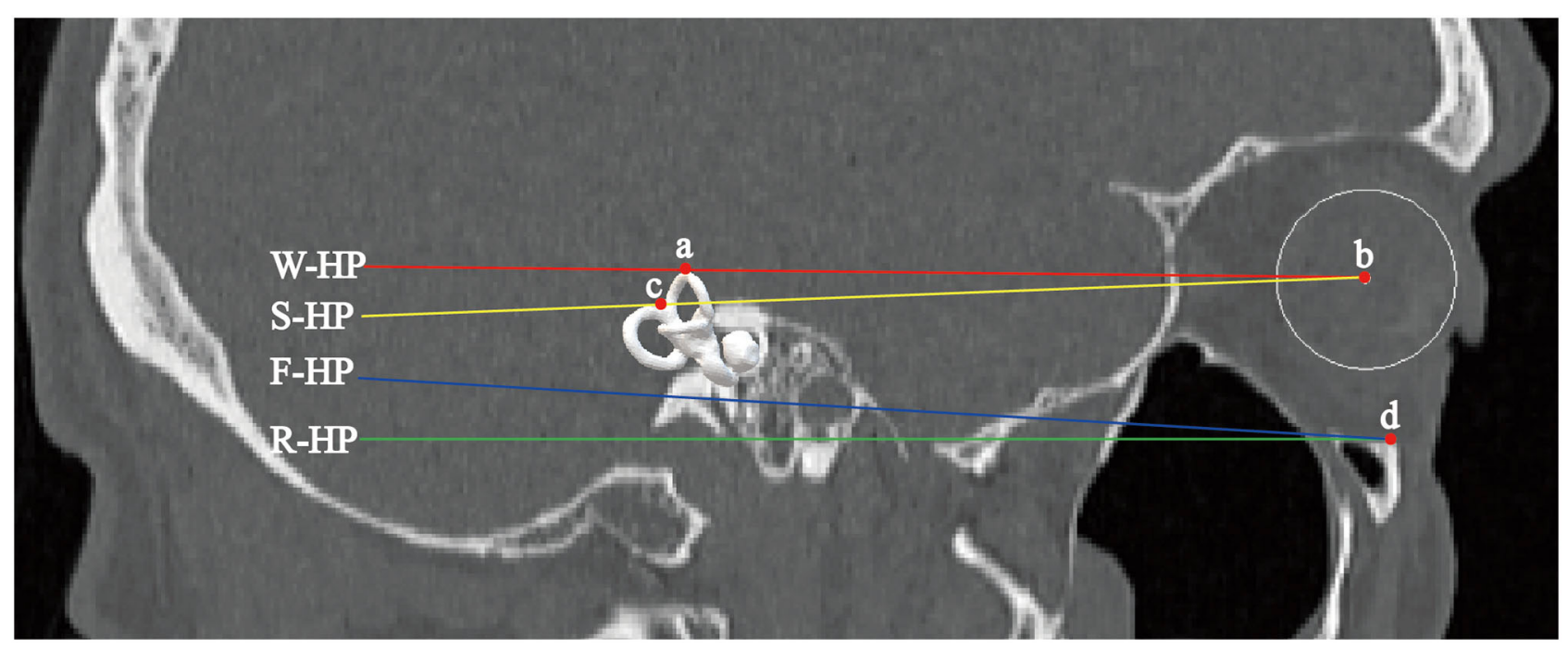

FIGURE 3 | Four different coordinate planes. Point a is the vertex of the bilateral superior semicircular canals. Point $b$ is the center of the eyeball. Point $c$ is the bifurcation of the common crus. Point d is the inferior margin of the orbit. W-HP, S-HP, F-HP, and R-HP refer to the horizontal planes of the W-X, Suzuki, Frankfurt, and Reid coordinate systems, respectively.

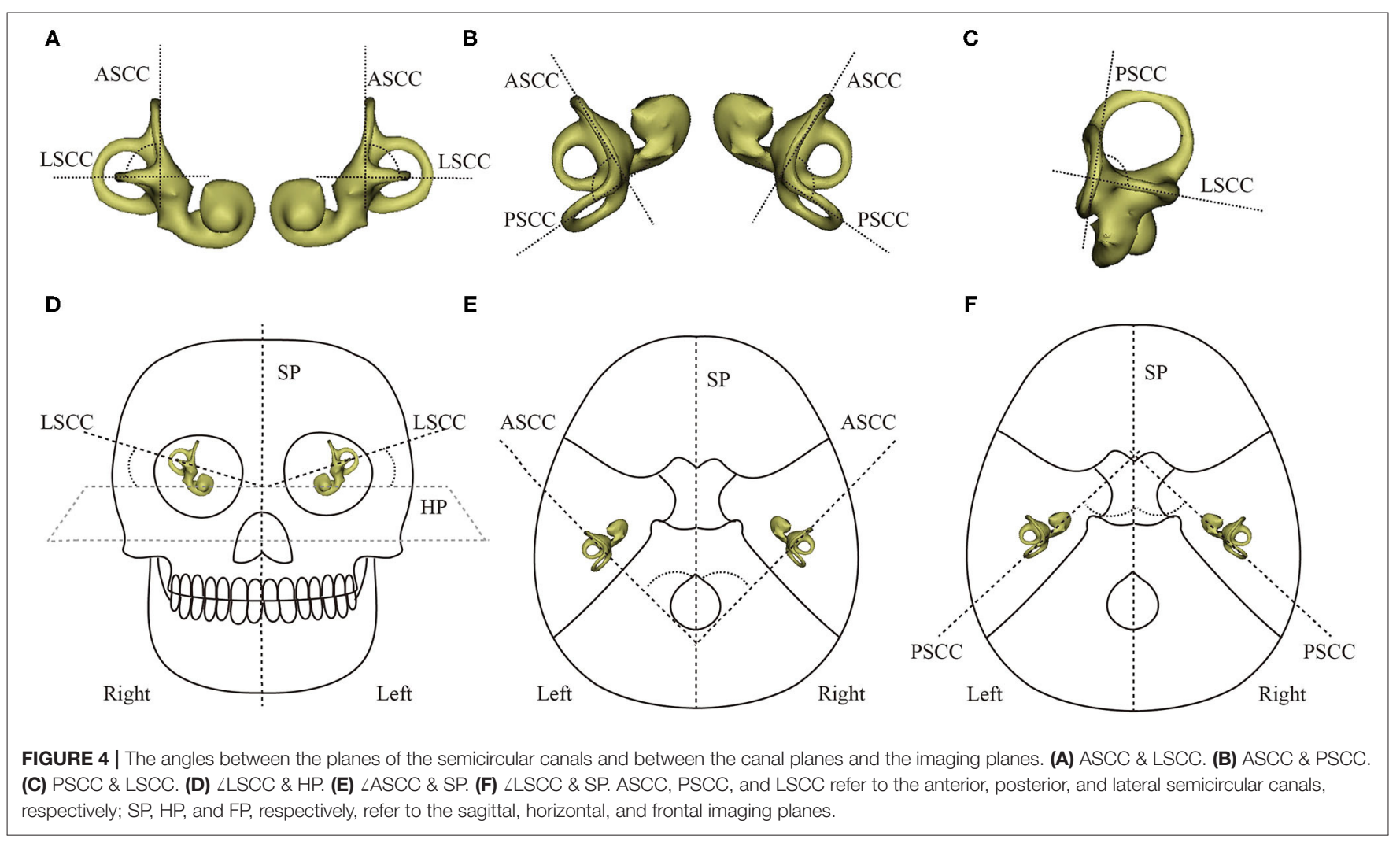

\section{Statistical Analysis}

Statistical analysis was performed using SPSS version 25.0 statistical software (SPSS Inc, IBM, Chicago, IL, USA). Data with normal or approximately normal distributions are represented as mean \pm SD. Differences between groups were measured using a $t$-test. All quoted $p$ values are two-sided and $p<0.05$ was considered statistically significant. 


\section{RESULTS}

\section{Angular Relationships Between Semicircular Canals}

The 3D model of the cochlea and semicircular canals is shown in Figures $\mathbf{5 A}, \mathbf{B}$. The centerline of the semicircular canals shows their morphological characteristics (Figures 5C,D). We measured the relative orientations of the semicircular canal planes (Table 1). There were no statistically significant differences between the angles measured using CT and MRI for any of the ipsilateral or contralateral semicircular canal planes. Therefore, the relative angles between the planes of semicircular canals measured using CT and MRI can be combined, yielding mean values for $\angle$ ASCC \& LSCC, $\angle$ ASCC \& PSCC, and $\angle$ LSCC \& PSCC of $84.67 \pm 5.76,94.21 \pm 3.81$, and $91.79 \pm 5.22$ degrees, respectively.

\section{Angles Between the LSCC and Four Different Coordinate Planes}

The angles between each of the horizontal reference planes and between each reference plane and the LSCC plane are shown in Table 2. The angle between the R-HP and $\mathrm{W}-\mathrm{X}$ plane was relatively small. There were no statistically significant differences between the values of $\angle \mathrm{LSCC} \& \mathrm{~W}$-HP and $\angle \mathrm{LSCC} \& \mathrm{R}-\mathrm{HP}$ $(t=1.62, p=0.11)$. However, there were statistical differences between $\angle \mathrm{LSCC} \& \mathrm{R}-\mathrm{HP}$ and $\angle \mathrm{LSCC} \& \mathrm{~F}-\mathrm{HP}(t=7.7, p<0.01)$, and between $\angle$ LSCC \& S-HP and $\angle$ LSCC \& R-HP $(t=-15.86$, $p<0.01)$. Therefore, $\mathrm{W}-\mathrm{HP}$ can be used in place of R-HP to establish the horizontal plane for MRI.

\section{Orientations of Semicircular Canals}

No statistically significant differences were observed between the orientations of the semicircular canals measured using Reid's coordinate system in CT and the $\mathrm{W}-\mathrm{X}$ coordinate system in MRI (Table 3). Therefore, the angles measured using CT and MRI can be combined. The angles between the ASCC plane and W-SP, W$\mathrm{HP}$, and $\mathrm{W}$-FP were thus $39.21 \pm 4.22,112.39 \pm 6.8$, and 124.60 \pm 3.69 degrees, respectively; the angles between the LSCC plane and SP, HP, and FP were 91.08 \pm 5.01, $15.64 \pm 3.92$, and $102.83 \pm$ 5.44 degrees, respectively; and the respective angles between the PSCC plane and SP, HP, and FP were $48.79 \pm 4.46,77.38 \pm 6.28$, and $134.53 \pm 3.80$ degrees. To confirm that the $\mathrm{W}-\mathrm{X}$ coordinate

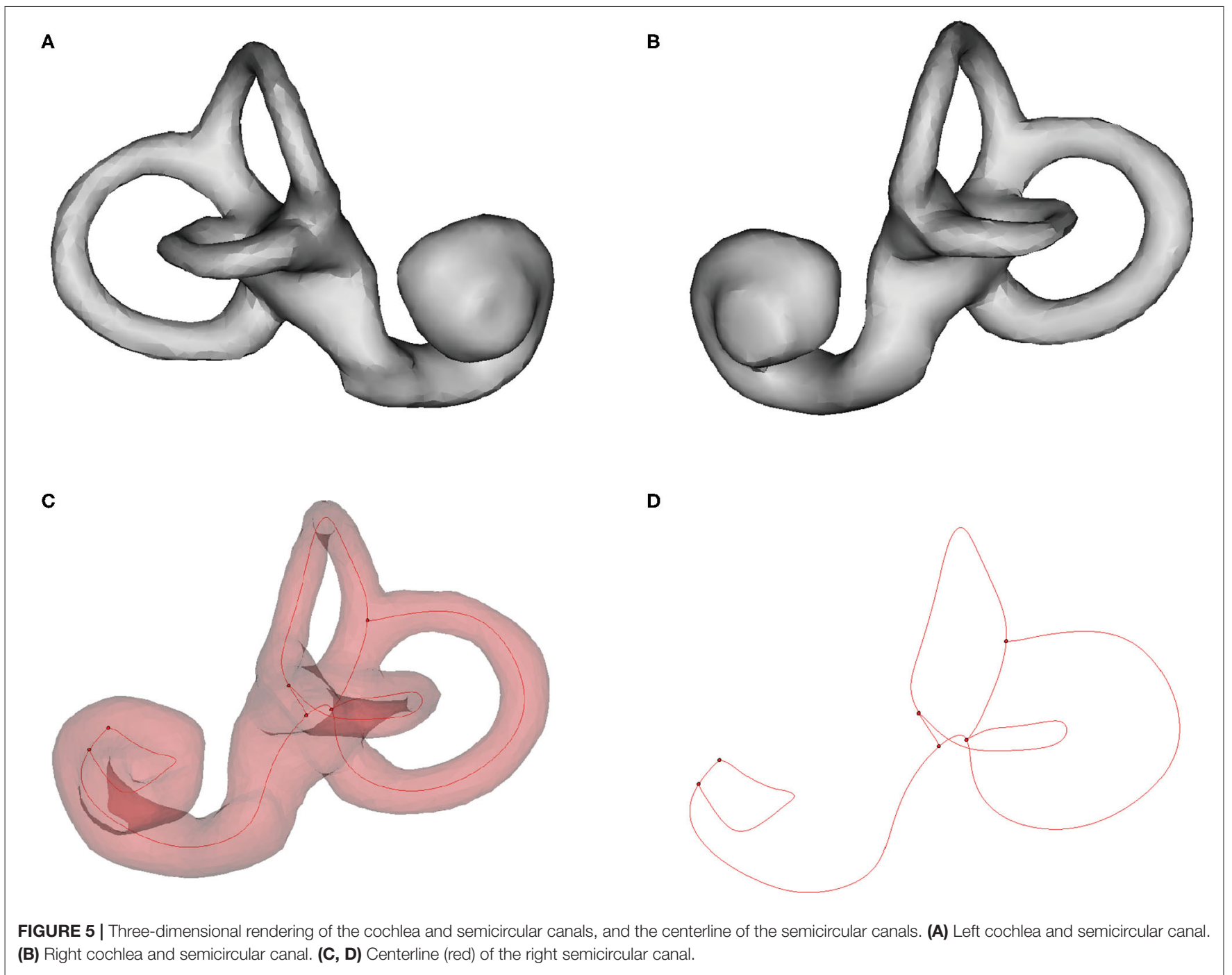


TABLE 1 | Angles (degrees) between the planes of the semicircular canals, measured using CT and MRI.

\begin{tabular}{|c|c|c|c|c|c|c|}
\hline \multirow[t]{2}{*}{ Angle } & \multicolumn{2}{|c|}{ CT $(n=78)$} & \multicolumn{2}{|c|}{ MRI $(n=53)$} & \multirow[b]{2}{*}{$\mathbf{t}$} & \multirow[b]{2}{*}{$p$} \\
\hline & Mean & $S D$ & Mean & $S D$ & & \\
\hline LASCC\& LSCC & 84.2176 & 6.05167 & 85.3321 & 5.29825 & -1.087 & 0.279 \\
\hline$\angle A S C C \&$ PSCC & 94.7569 & 3.81805 & 93.4272 & 3.69325 & 1.982 & 0.050 \\
\hline LLSCC \& PSCC & 92.0028 & 5.89758 & 91.4793 & 4.06055 & 0.602 & 0.548 \\
\hline$\angle R-A S C C \&$ L-ASCC & 75.095 & 7.58761 & 75.4098 & 6.85802 & -0.242 & 0.809 \\
\hline LL-LSCC \& R-LSCC & 167.9807 & 7.27765 & 165.9583 & 8.49 & 1.459 & 0.147 \\
\hline$\angle R-P S C C \&$ L-PSCC & 95.2038 & 7.59068 & 97.7854 & 6.97694 & -1.973 & 0.051 \\
\hline$\angle L-A S C C \& R-P S C C$ & 15.3473 & 5.73015 & 15.9285 & 4.85228 & -0.605 & 0.546 \\
\hline$\angle R-A S C C \&$ L-PSCC & 16.0313 & 5.45998 & 16.2188 & 4.51145 & -0.207 & 0.837 \\
\hline
\end{tabular}

ASCC, anterior semicircular canal; PSCC, posterior semicircular canal; LSCC, lateral semicircular canal. Prefixes "L-" and "R-" refer to canals on the left and right, respectively.

TABLE 2 | Angles (degrees) between the LSCC plane and the horizontal planes of the $\mathrm{W}-\mathrm{X}$ horizontal plane.

\begin{tabular}{lcc}
\hline Angles & Mean & SD \\
\hline LW-HP \& R-HP & 1.95 & 1.01 \\
LS-HP \& R-HP & 5.18 & 1.42 \\
LF-HP \& R-HP & 4.70 & 1.22 \\
LW-HP \& F-HP & 6.39 & 1.74 \\
LS-HP \& F-HP & 9.62 & 1.93 \\
LLSCC \&W-HP & 15.23 & 4.56 \\
LLSCC \& R-HP & 15.63 & 4.08 \\
LLSCC \& S-HP & 11.7 & 5.61 \\
LLSCC \& F-HP & 18.2 & 4.74 \\
\hline
\end{tabular}

(W-HP), Reid horizontal plane (R-HP), Frankfurt horizontal plane (F-HP), and Suzuki horizontal plane (S-HP) coordinate systems.

system can replace Reid's system, we analyzed data from patients who underwent both CT (Reid's system) and MRI (W-X system) and found no statistical differences between the angles measured using either system (Table 4).

\section{DISCUSSION}

We introduced a new $\mathrm{W}-\mathrm{X}$ coordinate system for MRI that uses the eyeballs and anterior semicircular canals as reference points. It is challenging to accurately fix the head position during CT and MRI examinations, so coordinate data in the file header of the DICOM image file may contain positioning errors. The $\mathrm{W}-\mathrm{X}$ coordinate system can solve this problem for stereotactic MRI analyses of cranial organs. Previous studies have commonly used Frankfurt and Reid coordinate systems, whose alignments usually rely on bony markers, such as the external auditory meatus and infraorbital ridge (12). However, it is difficult to accurately identify bony markers using MRI because air and bone yield low signal intensity. Suzuki et al. proposed an MRI coordinate system that uses the total foot bifurcation point of the semicircular canal and the eyeball midpoint to define the horizontal plane, S-HP (15). Aoki et al. found that R-HP lies very close to the S-HP (16). Conversely, we found a statistical difference between LLSCC \& S-HP and LLSCC \& R-HP. In addition, no statistical differences were found between the angles of semicircular canals measured using Reid's coordinate system (CT) and the $\mathrm{W}-\mathrm{X}$ coordinate system (MRI). Therefore, the W-X coordinate system can be used to analyze both CT and MRI data. In addition, all inner ear structures can be accurately mapped in the $\mathrm{W}-\mathrm{X}$ coordinate system because this system is uniquely defined for each subject. Additionally, the $\mathrm{W}-\mathrm{X}$ coordinate system can compensate for the structural complexity and measurement uncertainty of the midpoint of the lower edge of the orbit and the center of the external auditory canal in Reid's coordinate system.

MRI is more suitable than CT for routine anatomical research because it avoids exposure of subjects to radiation. In addition, it can visualize in detail the membrane labyrinth, peripheral nerve, and blood vessel structures from multiple angles and quantify the structure and volume of the inner ear (19). CT has long been used to assess the morphology of the temporal bone and assess the extent of lesions in the ossicular chain and bone labyrinth, but it cannot detect the membrane labyrinth (14). Membrane semicircular canals were reported to deviate from the bone semicircular canals by 2-6 degrees (13). However, our results showed no significant differences between CT and MRI images of these structures. The reason for this may be that the bone labyrinth is filled with internal and perilymph fluids but MRI mainly detects anatomical structures containing fluid (20).

The $\mathrm{W}-\mathrm{X}$ coordinate system provides a new means for measuring the orientations of the semicircular canals. Measuring the relative orientations and positions of the semicircular canals can be used for preoperative planning of surgical procedures, such as cochlear implantation $(21,22)$. It can provide advance knowledge of the location and degree of inner ear deformity and changes in labyrinthine shape, which can be used to develop personalized treatment plans. In addition, in vitro modeling of the semicircular canal requires precise measurement of the relative orientations and positions of the semicircular canals (23). 
TABLE 3 | Angles (degrees) between the planes of the semicircular canals and the sagittal plane (SP), horizontal plane (HP), and frontal plane (FP).

\begin{tabular}{|c|c|c|c|c|c|c|}
\hline \multirow[t]{2}{*}{ Angle } & \multicolumn{2}{|c|}{ CT $(n=78)$} & \multicolumn{2}{|c|}{ MRI $(n=53)$} & \multirow[b]{2}{*}{$\mathbf{t}$} & \multirow[b]{2}{*}{$p$} \\
\hline & Mean & SD & Mean & SD & & \\
\hline$\angle A S C C \& S P$ & 39.36 & 4.48 & 38.98 & 3.84 & 0.50 & 0.62 \\
\hline$\angle A S C C \& H P$ & 113.10 & 6.63 & 111.34 & 6.98 & 1.46 & 0.15 \\
\hline$\angle A S C C \& F P$ & 124.62 & 4.03 & 124.58 & 3.18 & 0.06 & 0.95 \\
\hline LLSCC \& SP & 90.76 & 4.84 & 91.54 & 5.28 & -0.87 & 0.38 \\
\hline$\angle \mathrm{LSCC} \& \mathrm{HP}$ & 15.59 & 3.47 & 15.72 & 4.53 & -0.19 & 0.85 \\
\hline$\angle L S C C \& F P$ & 102.49 & 5.52 & 103.35 & 5.34 & -0.88 & 0.38 \\
\hline$\angle \mathrm{PSCC} \& \mathrm{SP}$ & 48.42 & 4.71 & 49.34 & 4.04 & -1.17 & 0.24 \\
\hline$\angle P S C C \& H P$ & 77.64 & 6.38 & 77.01 & 6.18 & 0.56 & 0.58 \\
\hline$\angle P S C C \& F P$ & 134.11 & 3.94 & 135.15 & 3.53 & -1.53 & 0.13 \\
\hline
\end{tabular}

TABLE 4 | Angles (degrees) measured in the same patient between the planes of the semicircular canals and between the canal planes and the imaging planes.

\begin{tabular}{|c|c|c|c|c|c|c|}
\hline \multirow[t]{2}{*}{ Angle } & \multicolumn{2}{|c|}{ CT $(n=8)$} & \multicolumn{2}{|c|}{ MRI $(n=8)$} & \multirow[b]{2}{*}{$\mathbf{t}$} & \multirow[b]{2}{*}{$p$} \\
\hline & Mean & SD & Mean & SD & & \\
\hline$\angle A S C C \&$ LSCC & 86.93 & 2.94 & 85.68 & 5.62 & 0.55 & 0.60 \\
\hline$\angle A S C C \&$ PSCC & 94.86 & 2.66 & 93.22 & 4.25 & 2.20 & 0.06 \\
\hline LLSCC \& PSCC & 90.24 & 4.67 & 91.44 & 3.58 & -1.33 & 0.23 \\
\hline$\angle R-A S C C \&$ L-ASCC & 72.20 & 9.60 & 73.96 & 9.32 & -1.73 & 0.13 \\
\hline LL-LSCC \& R-LSCC & 168.48 & 7.12 & 166.61 & 7.13 & 0.59 & 0.57 \\
\hline$\angle R-P S C C \&$ L-PSCC & 99.31 & 8.70 & 101.73 & 6.41 & -1.93 & 0.10 \\
\hline LL-ASCC \& R-PSCC & 17.24 & 7.60 & 19.30 & 6.44 & -1.37 & 0.21 \\
\hline LR-ASCC \& L-PSCC & 16.61 & 8.61 & 16.59 & 6.13 & 0.01 & 0.99 \\
\hline$\angle A S C C \& S P$ & 36.31 & 4.97 & 37.11 & 4.68 & -1.65 & 0.14 \\
\hline$\angle A S C C \& H P$ & 98.89 & 5.82 & 98.38 & 6.84 & 0.39 & 0.71 \\
\hline$\angle A S C C \& F P$ & 124.17 & 3.43 & 124.97 & 2.14 & -1.11 & 0.30 \\
\hline$\angle L S C C \& S P$ & 90.83 & 4.30 & 89.98 & 4.06 & 0.43 & 0.68 \\
\hline$\angle \mathrm{LSCC} \& \mathrm{HP}$ & 15.20 & 3.12 & 14.41 & 3.81 & 0.98 & 0.36 \\
\hline$\angle L S C C \& F P$ & 103.37 & 3.28 & 102.18 & 4.77 & 0.65 & 0.54 \\
\hline$\angle \mathrm{PSCC} \& \mathrm{SP}$ & 49.86 & 4.30 & 51.02 & 3.17 & -1.90 & 0.10 \\
\hline$\angle P S C C \& H P$ & 78.24 & 6.94 & 76.89 & 7.08 & 2.27 & 0.06 \\
\hline$\angle P S C C \& F P$ & 136.58 & 3.99 & 137.01 & 2.48 & -0.64 & 0.54 \\
\hline
\end{tabular}

We found that the planes of adjacent semicircular canals were not perpendicular and the bilateral horizontal semicircular canals were not coplanar. To measure relative orientations of the semicircular canals, the plane of each semicircular canal needs to be obtained. However, the semicircular canals are not strictly planar, and the anterior canal showed the largest torsion compared with the others $(24,25)$. There exist no uniform criteria to best identify the approximate planes of the semicircular canals, and different methods may yield different results. Using the three points at the center of the cross-section of the canals (the ampulla and non-ampullated end, bifurcation at the vertical canals, and their approximate midpoint) to define a semicircular canal is the most common method, which can be easily performed in minimal time (15-18, 24, 26, 27). However, it cannot reflect the curvature of the semicircular canal and results are difficult to reproduce because of the inherent subjectivity. Direct measurement of the angle between the coordinate plane and the maximum cross-section of the semicircular canal (11, 28, 29) is simple but too subjective. Among them, the most objective approach is a multipoint fitting method, which defines the canal planes by calculation of 20-40 center positions in each cross-section of the canals using a least-squares method $(12,13,30)$ and can accurately describe the morphological structure of semicircular canals (25). In this study, we used third-party software to obtain the centerline and center points of the semicircular canals, reducing the error associated with manual point-picking. These center points were then used for the multipoint calculation of the canal planes. Therefore, the angles measured in this study are considered reliable.

Accurate structural information about the semicircular canals can aid the clinical diagnosis and treatment. Excluding patients with an inner ear deformity or superior semicircular canal dehiscence, we can use the orientations of semicircular canals to improve or develop a new BPPV treatment to reposition 
the otolith $(31,32)$. Approximately $10-20 \%$ of patients with suspected BPPV cannot be accurately diagnosed or effectively treated using routine procedures, such as the Dix-Hallpike, Epley, or Semont maneuvers $(9,10)$, which may be due to individual differences in the orientations of the semicircular canals and the speed of operation $(11,12,33)$. The Dix-Hallpike maneuver is performed by moving the patient from an upright to a supine position, with the head rotated by 45 degrees to one side, the neck extended by 20 degrees, and the affected ear facing down (31). We found that the average angle between the plane of the PSCC and the SP was 48.79 degrees, and the average angle between the LSCC and the HP was 15.64 degrees. This means that bowing the head by approximately 49 degrees and then rotating it to the right (left) by $\sim 16$ degrees will result in the coplanar alignment of the right (left) PSCC and the SP. Knowledge of the canal geometry is important for accurate stimulation of the LSCC during the roll test. This test assumes that the angle between the LSCC and the HP is 30 degrees, but our results indicate that this angle is 15.64 degrees. The latter angle is more conducive for moving the otolith from the PSCC into the vestibule. These findings suggest that accurate measurement of the semicircular canal geometry can be used to develop personalized BPPV treatment plans, especially for patients who have not responded to the repositioning therapy.

In summary, a new $\mathrm{W}$-X coordinate system was developed for MRI studies of the vestibular system and can reliably measure the orientations of the semicircular canals.

\section{DATA AVAILABILITY STATEMENT}

The raw data supporting the conclusions of this article will be made available by the authors, without undue reservation.

\section{REFERENCES}

1. Hülse R, Biesdorf A, Hörmann K, Stuck B, Erhart M, Hülse M, et al. Peripheral vestibular disorders: an epidemiologic survey in 70 million individuals. Eur Acad Otol Neurotol. (2019) 40:88-95. doi: 10.1097/MAO.0000000000002013

2. Strupp M, Dlugaiczyk J, Ertl-Wagner BB, Rujescu D, Westhofen M, Dieterich M. Vestibular disorders: diagnosis, new classification and treatment. Dtsch Arztebl Int. (2020) 117:300-10. doi: 10.3238/arztebl.2020.0300

3. Cheng Z, Gu Y. Vestibular system and self-motion. Front Cell Neurosci. (2018) 12:456. doi: 10.3389/fncel.2018.00456

4. Asadi H, Mohamed S, Lim CP, Nahavandi S, Nalivaiko E. Semicircular canal modeling in human perception. Rev Neurosci. (2017) 28:53749. doi: 10.1515/revneuro-2016-0058

5. Yang A, Hullar TE. Relationship of semicircular canal size to vestibularnerve afferent sensitivity in mammals. J Neurophysiol. (2007) 98:3197205. doi: 10.1152/jn.00798.2007

6. Berlin JC, Kirk EC, Rowe TB. Functional implications of ubiquitous semicircular canal non-orthogonality in mammals. PLoS ONE. (2013) 8:246. doi: 10.1371/journal.pone.0079585

7. Malinzak MD, Kay RF, Hullar TE. Locomotor head movements and semicircular canal morphology in primates. Proc Natl Acad Sci U S A. (2012) 109:17914-9. doi: 10.1073/pnas.1206139109

8. Ifediba MA, Rajguru SM, Hullar TE, Rabbitt RD. The role of 3-canal biomechanics in angular motion transduction by the human vestibular labyrinth. Ann Biomed Eng. (2007) 35:124763. doi: 10.1007/s10439-007-9277-y

\section{ETHICS STATEMENT}

The studies involving human participants were reviewed and approved by the First Affiliated Hospital of Guangzhou Medical University Institutional Review Board. Written informed consent from the patients/ participants or patients/participants' legal guardian/next of kin was not required to participate in this study in accordance with the national legislation and the institutional requirements.

\section{AUTHOR CONTRIBUTIONS}

WL, XZ, and LS: conception and design. XZ: administrative support. WL, GC, JX, and TL: provision of study materials or patient data. WL, GC, JX, TL, CZ, XL, WL, and LS: collection and assembly of data. WL, GC, JX, TL, and LS: data analysis and interpretation. All the authors have written and approved the final manuscript.

\section{FUNDING}

This work was supported by the Science and Technology Planning Project of Guangdong Province (grant 2016A020220019), the Upper Respiratory Disease Innovation and Transformation Platform Construction Project of Guangdong Provincial, and the High-level Construction Project of Guangzhou Medical University.

\section{ACKNOWLEDGMENTS}

We thank Liwen Bianji (Edanz) (www.liwenbianji.cn/ac) for editing the language of a draft of this manuscript.

9. Anagnostou E, Kouzi I, Spengos K. Diagnosis and treatment of anteriorcanal benign paroxysmal positional vertigo: a systematic review. J Clin Neurol. (2007) 11:262-7. doi: 10.3988/jen.2015.11.3.262

10. Cromwell C, Tyler J, Nobbs R, Hockaday A, Donnelly S, Clendaniel R. The necessity for post-maneuver restrictions in the treatment of benign paroxysmal positional vertigo: an updated meta-analysis of the literature. Otol Neurotol. (2018) 39:671-9. doi: 10.1097/MAO.00000000000 01798

11. Tang X, Tang J, Gong L, Ke R, Tan S, Tang A. Adverse effects of semicircular circle angles variation on Epley repositioning procedure: a study on reconstruction of Micro-CT images 3D. Acta Otolaryngol. (2020) 140:99104. doi: 10.1080/00016489.2019.1699953

12. Blanks RHI, Curthoys IS, Markham CH. Planar relationships of the semicircular canals in man. Acta Otolaryngol. (1975) 80:185-96. doi: 10.3109/00016487509121318

13. Hashimoto $\mathrm{S}$, Naganuma $\mathrm{H}$, Tokumasu $\mathrm{K}$, Itoh $\mathrm{A}$, Okamoto $\mathrm{M}$. Three-dimensional reconstruction of the human semicircular canals and measurement of each membranous canal plane defined by Reid's stereotactic coordinate. Ann Otol Rhinol Laryngol. (2005) 114:934-8. doi: 10.1177/000348940511401207

14. Hullar TE, Williams CD. Geometry of the semicircular canals of the chinchilla (Chinchilla laniger). Hear Res. (2006) 213:1724. doi: 10.1016/j.heares.2005.11.009

15. Suzuki K, Masukawa A, Aoki S, Arai Y, Ueno E. A new coordinate system for cranial organs using magnetic resonance imaging. Acta Otolaryngol. (2010) 130:568-75. doi: 10.3109/00016480903317473 
16. Aoki S, Takei Y, Suzuki K, Masukawa A, Arai Y. Planer orientation of the bilateral semicircular canals in dizzy patients. Auris Nasus Larynx. (2012) 39:451-4. doi: 10.1016/j.anl.2011.07.020

17. Lyu HY, Chen KG, Yin DM, Hong J, Yang L, Zhang TY, et al. The age-related orientational changes of human semicircular canals. Clin Exp Otorhinolaryngol. (2016) 9:109-15. doi: 10.21053/ceo.2014.02012

18. Kim DK, Kim DR, Jeong SH, Kim GJ, Chang KH, Jun BC. Analysis of the coplanarity of functional pairs of semicircular canals using three-dimensional images reconstructed from temporal bone magnetic resonance imaging. $J$ Laryngol Otol. (2015) 129:430-4. doi: 10.1017/S0022215115000201

19. Wu HB, Yuan HS, Ma F, Zhao Q. Comparison of FSE T2 W PROPELLER and 3D-FIESTA of $3 \mathrm{~T}$ MR for the internal auditory canal. Clin Imaging. (2017) 45:30-3. doi: 10.1016/j.clinimag.2016.09.001

20. Seemann MD, Beltle J, Heuschmid M, Löwenheim H, Graf H, Claussen CD. Image fusion of CT and MRI for the visualization of the auditory and vestibular system. Eur J Med Res. (2005) 10:47-55.

21. Jia H, Torres R, Nguyen Y, De Seta D, Ferrary E, Wu H, et al. Intraoperative conebeam CT for assessment of intracochlear positioning of electrode arrays in adult recipients of cochlear implants. Am J Neuroradiol. (2018) 39:76874. doi: 10.3174/ajnr.A5567

22. Deshpande AS, Soares BP, Todd NW. Spatial orientation of the adult cochlea: rotation, tilt, and angle theta 3. Surg Radiol Anat. (2018) 40:697704. doi: 10.1007/s00276-018-2017-1

23. Obrist $\mathrm{D}$, Hegemann S, Kronenberg $\mathrm{D}$, Häuselmann $\mathrm{O}$, Rösgen $\mathrm{T}$. In vitro model of a semicircular canal: design and validation of the model and its use for the study of canalithiasis. J Biomech. (2010) 43:120814. doi: 10.1016/j.jbiomech.2009.11.027

24. Sato H, Sando I, Takahashi H, Fujita S. Torsion of the human semicircular canals and its influence on their angular relationships. Acta Otolaryngol. (1993) 113:171-5. doi: 10.3109/00016489309135787

25. Bradshaw AP, Curthoys IS, Todd MJ, Magnussen JS, Taubman DS, Aw ST, et al. A mathematical model of human semicircular canal geometry: a new basis for interpreting vestibular physiology. J Assoc Res Otolaryngol. (2010) 11:145-59. doi: 10.1007/s10162-009-0195-6

26. El Khoury M, Braga J, Dumoncel J, Nancy J, Esclassan R, Vaysse F. The human semicircular canals orientation is more similar to the bonobos than to the chimpanzees. PLoS ONE. (2014) 9:e93824. doi: 10.1371/journal.pone.0093824

27. Mejdoubi M, Dedouit F, Mokrane FZ, Telmon N. Semicircular canal angulation during fetal life: a computed tomography study of 54 human fetuses. Otol Neurotol. (2015) 36:7014. doi: 10.1097/MAO.0000000000000640
28. Della Santina CC, Potyagaylo V, Migliaccio AA, Minor LB, Carey JP. Orientation of human semicircular canals measured by three-dimensional multiplanar CT reconstruction. J Assoc Res Otolaryngol. (2005) 6:191206. doi: 10.1007/s10162-005-0003-X

29. Lee JY, Shin KJ, Kim JN, Yoo JY, Song WC, Koh KS, et al. Morphometric study of the semicircular canals using micro-CT images in threedimensional reconstruction. Anat Rec. (2013) 296:834-9. doi: 10.1002/ar. 22664

30. Cox PG, Jeffery N. Geometry of the semicircular canals and extraocular muscles in rodents, lagomorphs, felids and modern humans. J Anat. (2008) 213:583-96. doi: 10.1111/j.1469-7580.2008.00983.x

31. Bhattacharyya N, Gubbels SP, Schwartz SR, Edlow JA, El-Kashlan $\mathrm{H}$, Fife $\mathrm{T}$, et al. Clinical practice guideline: benign paroxysmal positional vertigo (Update). Otolaryngol Head Neck Surg. (2017) 156:S1-47. doi: 10.1177/0194599816689667

32. Yang XK, Zheng YY, Yang XG. Theoretical observation on diagnosis maneuver for benign paroxysmal positional vertigo. Acta Otolaryngol. (2017) 137:56771. doi: 10.1080/00016489.2016.1271451

33. Hwang M, Kim SH, Kang KW, Lee D, Lee SY, Kim MK, et al. Canalith repositioning in apogeotropic horizontal canal benign paroxysmal positional vertigo: do we need faster maneuvering? J Neurol Sci. (2015) 358:1837. doi: 10.1016/j.jns.2015.08.1534

Conflict of Interest: The authors declare that the research was conducted in the absence of any commercial or financial relationships that could be construed as a potential conflict of interest.

Publisher's Note: All claims expressed in this article are solely those of the authors and do not necessarily represent those of their affiliated organizations, or those of the publisher, the editors and the reviewers. Any product that may be evaluated in this article, or claim that may be made by its manufacturer, is not guaranteed or endorsed by the publisher.

Copyright (c) 2022 Liu, Chen, Xie, Liang, Zhang, Liao, Liao, Song and Zhang. This is an open-access article distributed under the terms of the Creative Commons Attribution License (CC BY). The use, distribution or reproduction in other forums is permitted, provided the original author(s) and the copyright owner(s) are credited and that the original publication in this journal is cited, in accordance with accepted academic practice. No use, distribution or reproduction is permitted which does not comply with these terms. 